Draft VERSION MAY 14, 2018

Preprint typeset using IATEX style emulateapj v. 11/26/04

\title{
THE DEEP3 GALAXY REDSHIFT SURVEY: KECK/DEIMOS SPECTROSCOPY IN THE GOODS-N FIELD*
}

\author{
Michael C. Cooper ${ }^{1, \dagger, 2, \ddagger}$ James A. Aird $^{3}$. Alison L. Coil ${ }^{3, \S}$, Marc Davis $^{4,5}$, S. M. Faber ${ }^{6}$, Stéphanie Juneau ${ }^{2}$, \\ Jennifer M. Lotz ${ }^{7}$, Kirpal Nandra $^{8}$, Jeffrey A. Newman ${ }^{9}$, Christopher N. A. Willmer ${ }^{2}$, Rendin Yan $^{10,11}$ \\ Draft version May 14, 2018
}

\begin{abstract}
We present the results of spectroscopic observations in the GOODS-N field completed using DEIMOS on the Keck II telescope as part of the DEEP3 Galaxy Redshift Survey. Observations of 370 unique targets down to a limiting magnitude of $R_{\mathrm{AB}}=24.4$ yielded 156 secure redshifts. In addition to redshift information, we provide sky-subtracted one- and two-dimensional spectra of each target. Observations were conducted following the procedures of the Team Keck Redshift Survey (TKRS), thereby producing spectra that augment the TKRS sample while maintaining the uniformity of its spectral database.

Subject headings: galaxies: distances and redshifts; catalogs; surveys
\end{abstract}

\section{INTRODUCTION}

Due in large part to the Great Observatories Origins Deep Survey (GOODS, Giavalisco et al. 2004), the GOODS-N field $\left(\alpha=12^{\mathrm{h}} 36^{\mathrm{m}} 55^{\mathrm{s}}, \delta=+62^{\circ} 14^{\mathrm{m}} 15^{\mathrm{s}}\right)$ has become one of the most well-studied extragalactic fields in the sky with existing observations among the deepest at a broad range of wavelengths (e.g., Alexander et al. 2003; Morrison et al. 2010; Elbaz et al. in prep). In the coming years, this status as one of the very deepest multiwavelength survey fields will be further cemented

\footnotetext{
* The data presented herein were obtained at the W. M. Keck Observatory, which is operated as a scientific partnership among the California Institute of Technology, the University of California and the National Aeronautics and Space Administration. The Observatory was made possible by the generous financial support of the W. M. Keck Foundation.

${ }^{1}$ Center for Galaxy Evolution, Department of Physics and Astronomy, University of California, Irvine, 4129 Frederick Reines Hall, Irvine, CA 92697, USA; m.cooper@uci.edu

$\dagger$ Hubble Fellow

2 Steward Observatory, University of Arizona, 933 N. Cherry Avenue, Tucson, AZ 85721, USA; sjuneau@as.arizona.edu, cnaw@as.arizona.edu

$¥$ Spitzer Fellow

3 Center for Astrophysics and Space Sciences, University of California, San Diego, 9500 Gilman Drive, MC 0424, San Diego, CA 92093, USA; acoil@ucsd.edu, jaird@ucsd.edu

$\S$ Alfred P. Sloan Foundation Fellow

${ }^{4}$ Department of Astronomy, University of California, Berkeley, Mail Code 3411, Berkeley, CA 94720, USA; marc@astro.berkeley.edu

${ }^{5}$ Department of Physics, University of California, Berkeley, Mail Code 7300, Berkeley, CA 94720, USA

${ }^{6} \mathrm{UCO} /$ Lick Observatory and Department of Astronomy and Astrophysics, University of California, Santa Cruz, 1156 High Street, Santa Cruz, CA 95064, USA; faber@ucolick.org, koo@ucolick.org,raja@ucolick.org

${ }^{7}$ Space Telescope Science Institute, 3700 San Martin Drive, Baltimore, MD 21218, USA; lotz@stsci.edu

${ }^{8}$ Max-Planck-Institut für Extraterrestrische Physik (MPE), Postfach 1312, Giessenbachstrasse 1, D-85741 Garching, Germany; knandra@mpe.mpg.de

${ }^{9}$ Department of Physics and Astronomy, University of Pittsburgh, 401-C Allen Hall, 3941 O'Hara Street, Pittsbrugh, PA 15260, USA; janewman@pitt.edu

10 Department of Astronomy and Astrophysics, University of Toronto, 50 St. George Street, Toronto, ON M5S 3H4, Canada

${ }^{11}$ Center for Cosmology and Particle Physics, Department of Physics, New York University, 4 Washington Place, New York, NY 10003, USA; renbin@nyu.edu
}

by the ongoing and upcoming extremely-deep observations with Spitzer/IRAC and HST/WFC3-IR as part of the Spitzer Extended Deep Survey (SEDS, PI G. Fazio) and the Cosmic Assembly Near-IR Deep Extragalactic Legacy Survey (CANDELS, PIs S. Faber \& H. Ferguson), respectively.

Given the large commitment of telescope time from both space- and ground-based facilities devoted to imaging the GOODS-N field, spectroscopic observations in this field possess a significant legacy value. For instance, spectroscopic redshifts dramatically improve the constraints inferred from imaging alone, allowing rest-frame quantities to be derived with increased precision. Furthermore, only through spectroscopy can assorted spectral and dynamical properties (such as the strengths and velocity widths of emission and absorption lines) be measured.

Recognizing the potential legacy value of spectroscopic observations in the GOODS-N field, the Team Keck Redshift Survey (TKRS, Wirth et al. 2004) utilized the DEep Imaging Multi-Object Spectrograph (DEIMOS, Faber et al. 2003) on the Keck II telescope to create a publiclyavailable redshift catalog and uniform spectral database across the entire area imaged with $H S T$ /ACS by the GOODS Team. Altogether, the TKRS observed nearly 3000 sources, yielding secure spectroscopic redshifts for $\sim 1500$ objects and enabling numerous studies of galaxy evolution and cosmology (e.g., Kobulnicky \& Kewley 2004; Weiner et al. 2006; Riess et al. 2007; Juneau et al. 2010).

In an effort to augment the value of the existing TKRS dataset, we present observations of 370 unique sources in the GOODS-N field (a > 10\% increase to the TKRS sample size), collected as part of the DEEP3 Galaxy Redshift Survey (Cooper et al. 2011, in prep) and using the same instrument and observation methods as the TKRS. The DEEP3 survey is an ongoing spectroscopic effort designed to leverage the vast amounts of multiwavelength data in another prime deep extragalactic field, the Extended Groth Strip (EGS). Once completed, DEEP3 will yield Keck/DEIMOS spectra of $\gtrsim 7500$ sources at $z<2$, which when combined with TKRS and this work will create an extensive spectral database that is both uniform and publicly-available. In Sections 2 and 
3 , we describe the design, execution, and reduction of our Keck/DEIMOS observations in GOODS-N, with the data products presented in Section 4.

\section{TARGET SELECTION AND SLIT MASK DESIGN}

The 391 spectra presented herein were distributed across 4 Keck/DEIMOS slit masks, designed using the DSIMULATOR software (part of the DEIMOS IRAF package). In order to match the TKRS, targets were selected down to a limiting magnitude of $R_{\mathrm{AB}}=24.4$. However, while DEIMOS $R$-band imaging was employed to select the TKRS sample, our spectroscopic targets were drawn from the optical imaging catalogs of Capak et al. (2004).

Furthermore, our observations cover a moderately wider area of sky than those of the TKRS, extending slightly beyond the borders of the GOODS HST/ACS footprint (see Figure 1). Like other deep observations in GOODS-N from Chandra (Alexander et al. 2003) and the VLA (Morrison et al. 2010), the deep Spitzer/MIPS data from the Far-Infrared Deep Extragalactic Legacy (FIDEL) Survey (PI M. Dickinson), which incorporates observations from the Spitzer GO program of Frayer et al. (2006), cover a larger area than the HST/ACS imaging. Our slit masks were positioned, in part, to target sources detected at $24 \mu \mathrm{m}$ and/or $70 \mu \mathrm{m}$ by the FIDEL Survey, but not observed by the TKRS or as part of the spectroscopic observations of Cowie et al. (2004) and Barger et al. (2008). The optical counterparts to the Spitzer/MIPS sources were manually selected as part of the slit mask design, using the MIPS catalogs of Magnelli et al. (2011), while sources with existing redshifts in the TKRS and Barger et al. (2008) catalogs were downweighted.

Finally, the 2 Ms Chandra data in the GOODS-N field were reprocessed following the methodology of Laird et al. (2009), with optical counterparts to the x-ray point sources being identified in the Capak et al. (2004) imaging using the likelihood ratio technique of Aird et al. (2010). Any x-ray sources lacking an existing spectroscopic redshift in the literature, were prioritized whenever possible. The total number of unique targets on the 4 slit masks totals 370 , with 21 objects appearing on 2 slit masks (i.e., we obtained 391 spectra of 370 unique targets). Note that objects targeted specifically as Spitzer or Chandra sources are identified accordingly in the redshift catalog (see Table 2).

In designing the DEIMOS slit masks, slits were tilted up to \pm 30 degrees relative to the mask position angle to align with the major axis of elongated targets (i.e., targets with an ellipticity $e \equiv 1-(b / a) \geq 0.3)$. When possible, an object's ellipticity and the orientation of its major axis were estimated from the GOODS HST/ACS imaging using the ELLIPTICITY and THETA_IMAGE values in the $i_{\mathrm{F} 775 \mathrm{~W}}$-band imaging catalog (version $\mathrm{r} 2.0 \mathrm{z}$ ). For sources outside of the GOODS HST/ACS footprint, the elongation and orientation of an object were measured from the $R$-band imaging of Capak et al. (2004) using the SExtractor software package (Bertin \& Arnouts 1996). For non-elongated sources (i.e., $e<0.3$ ), slits were tilted \pm 5 degrees relative to the mask position angle so as to provide improved wavelength sampling of the sky background.

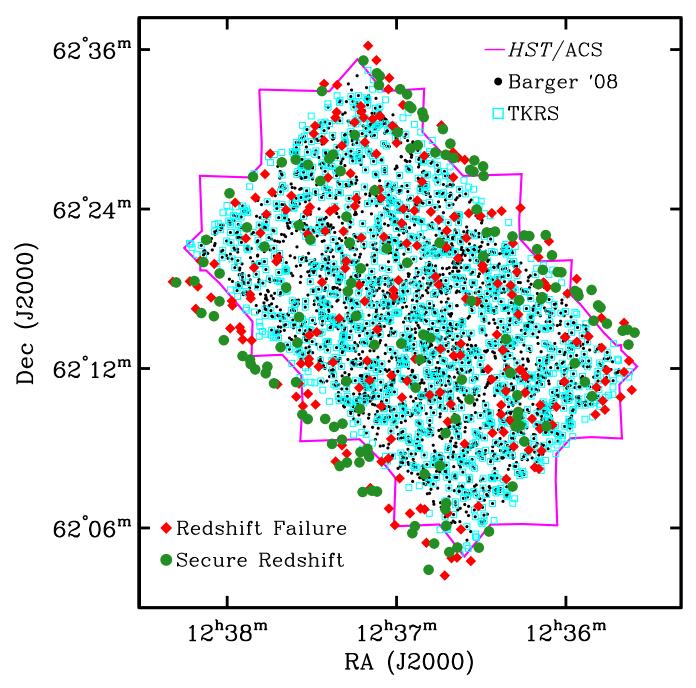

FIG. 1. - The distribution of our Keck/DEIMOS targets on the sky. The cyan squares and black points show the location of sources with redshifts in the TKRS (Wirth et al. 2004) and Barger et al. (2008) samples, respectively. The filled red diamonds and green circles denote the Keck/DEIMOS targets presented herein, with the green (versus red) color distinguishing those sources for which we succeeded (versus failed) in obtaining a secure redshift. Note that our target population extends beyond the GOODS HST/ACS footprint (denoted by the magenta outline), including previously unobserved Spitzer sources.

\section{OBSERVATIONS AND DATA REDUCTION}

Spectroscopic observations using DEIMOS were completed during the Spring of 2009 and 2010 as detailed in Table 1. Observations utilized the 600 lines $\mathrm{mm}^{-1}$ grating blazed at $7500 \AA$ and tilted to a central wavelength of $7200 \AA$, which yields a nominal spectral coverage of $4600-9800 \AA$ at a resolution $(\mathrm{FWHM})$ of $\sim 3.5 \AA$. The GG455 order-blocking filter was employed to eliminate all flux blueward of $4550 \AA$. Each slit mask was observed for a total integration time of $\sim 3600 \mathrm{sec}$, divided into (at least) 3 individual $\sim 1200 \mathrm{sec}$ integrations (with no dithering performed) to facilitate the rejection of cosmic rays - see Table 1 for details regarding the total integration times. Standard calibration frames, consisting of three flat-field frames utilizing the internal quartz lamp and a single arc lamp spectrum (using $\mathrm{Kr}, \mathrm{Ar}$, Ne, and $\mathrm{Xe}$ ), were collected for each mask with the DEIMOS flexure compensation system ensuring that the instrument light path for all calibration images matched the science images to better than \pm 0.25 pixels.

The transparency and seeing conditions varied from poor to fair, resuting in data of limited quality. The typical seeing varied from $\sim 0.6^{\prime \prime}$ to $1^{\prime \prime}$ with variable cloud cover and high humidity at times. In addition, our observations of mask 20 were affected by intermittent dropout of the 1B CCD amplifier in DEIMOS. This issue, which impacted two of the four exposures for mask 20, caused all data on one-eighth of the CCD array (i.e., the blue half of the spectrum for $\sim 25 \%$ of all objects) to be lost. As a result, the effective exposure time for the blue half of the spectra in slits 0-1, 34-38, 40-41, and 44-62 of mask 20 (28 slits in total) is a factor of 2 lower than that for the corresponding red half. The resulting variation in integration time with wavelength for each of these slits results in discontinuities in the associated object spec- 
tra. The spectra for a fraction of these objects still yield secure redshifts (i.e., present multiple identifiable spectral features, with a resolved [OІІ] $\lambda \lambda 3726,3729 \AA$ doublet counting as two features); however, care must be taken in any studies utilizing information from the blue halves of the spectra.

The DEIMOS spectroscopic observations were reduced using the fully-automated DEEP2/DEIMOS data reduction pipeline (Newman et al., in prep; Cooper et al., in prep) developed as part of the DEEP2 Galaxy Redshift Survey (Davis et al. 2003; Newman et al., in prep). ${ }^{16}$ Redshifts were measured from the reduced spectra using additional software developed as part of the DEEP2 Galaxy Redshift Survey. All spectra were visually inspected, with a quality code $(Q)$ assigned corresponding to the accuracy of the redshift value $-Q=-1,3,4$ denote secure redshifts, with $Q=-1$ corresponding to stellar sources and $Q=3,4$ denoting secure galaxy redshifts (see Table 2). For detailed descriptions of the reduction pipeline, redshift measurement code, and quality assignment process refer to Wirth et al. (2004), Davis et al. (2007), and Newman et al. (in prep).

\section{DATA}

The redshift measurements resulting from our Keck/DEIMOS spectroscopy are presented in Table 2, a subset of which is listed herein. The entirety of Table 2 appears in the electronic version of the Journal and also on the DEEP Team website. ${ }^{17}$ Sky-subtracted onedimensional and two-dimensional spectra corresponding to each entry in the redshift catalog are also available at the same website. Note that a redshift is only included when classified as being secure, $(Q=-1,3,4)$. The total number of secure redshifts in the sample is 156 out of 370 total, unique targets. In Figure 2, we show the redshift distribution for this sample. The low redshift success rate is largely due to the poor conditions on Mauna Kea during the observations.

Matching our catalog to those of Barger et al. (2008) and Wirth et al. (2004), we find 34 of our targets have a redshift published as part of these existing data sets; 5 of 34 are matched to both catalogs. For 22 of these 34 objects, we measure a secure redshift from our DEIMOS spectroscopy. While this sample is quite small, the agreement between our redshifts and those of Barger et al. (2008) and Wirth et al. (2004) is excellent. We find a median offset of $|\Delta z| \sim 70 \mathrm{~km} \mathrm{~s}^{-1}$ and a maximum difference of $530 \mathrm{~km} \mathrm{~s}^{-1}$.

The new redshifts presented here should significantly enhance studies of galaxy evolution and cosmology in the GOODS-N field. Our sample expands upon the work of the Team Keck Redshift Survey, increasing the size of the existing TKRS redshift and spectral data sets by approximately $10 \%$. In addition, our observations broaden the area covered by the TKRS to extend beyond the GOODS $H S T /$ ACS footprint, allowing us to target a greater number of relatively rare sources.

In particular, we specifically targeted Spitzer/MIPS and Chandra sources not previously observed by TKRS and other spectroscopic efforts in the field (e.g., Lowenthal et al. 1997; Phillips et al. 1997; Cohen et al. 2000;

\footnotetext{
16 http://deep.berkeley.edu/spec2d/

17 http://deep.berkeley.edu/GOODSN
}

Dawson et al. 2001; Treu et al. 2005; Reddy et al. 2006; Barger et al. 2008). Within the FIDEL Survey's Spitzer/MIPS $70 \mu \mathrm{m}$ photometric catalog for GOODS$\mathrm{N}$, there are less than 100 sources with a 5- $\sigma$ detection down to $3.2 \mathrm{mJy}$ (Magnelli et al. 2011). The relatively small number of these sources puts a premium on spectroscopic follow-up, including those located outside of the area imaged with $H S T /$ ACS. The $70 \mu \mathrm{m}$ observations conducted as part of the FIDEL Survey are the deepest in the sky, allowing significant numbers of star-forming galaxies and active galactic nuclei to be detected out to intermediate redshift at rest-frame wavelengths that are dramatically less impacted by aromatic and silicate emission than those normally probed by Spitzer/MIPS $24 \mu \mathrm{m}$ observations. With accompanying redshift information from spectroscopic follow-up such as presented here, these deep far-infrared data provide a unique constaint on the cosmic star-formation history at intermediate redshift (e.g., Magnelli et al. 2009).

Finally, by extending beyond the HST/ACS footprint (i.e., the field surveyed by TKRS and Barger et al. 2008), this work has taken an initial step towards expanding the area over which galaxy overdensity (or "environment") can be measured in the GOODS-N field. The finite area of sky covered by a survey introduces geometric distortions - or edge effects - which bias environment measures near borders (or holes) in the survey field, generally leading to an underestimate of the local overdensity (Cooper et al. 2005, 2006). To minimize the impact of these edge effects on studies of galaxy environment, galaxies near the edge of the survey field (e.g., within a projected distance of 1-2 $h^{-1}$ comoving Mpc of an edge) are often excluded from any analysis. Thus, the data presented herein, when combined with additional spectroscopic observations that similarly broaden the survey field, will allow the environment of galaxies at intermediate redshift to be accurately computed across the entire $H S T /$ ACS area in the GOODS-N field, enabling unique studies of small-scale clustering in one of the most wellstudied extragalactic fields in the sky.

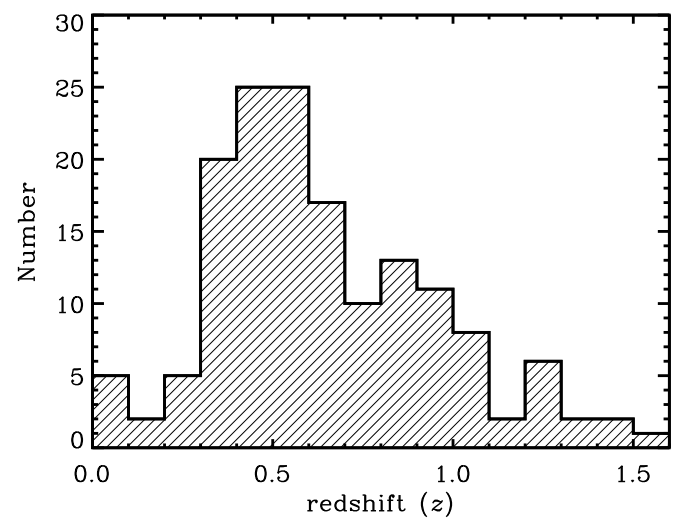

FIG. 2.- The distribution of the 156 unique, secure $(Q=$ $-1,3,4)$ redshifts measured from the Keck/DEIMOS spectroscopy.

Support for this work was provided by NASA through the Spitzer Space Telescope Fellowship Program. 
TABLE 1

Slit Mask Observation Information

\begin{tabular}{cccccccc}
\hline $\begin{array}{c}\text { Slit Mask } \\
\text { Number }\end{array}$ & $\begin{array}{c}\text { Observation Date } \\
\text { (UT) }\end{array}$ & $\alpha(\mathrm{J} 2000)^{\mathrm{a}}$ & $\delta(\mathrm{J} 2000)$ & $\begin{array}{c}\text { P.A. }{ }^{\mathrm{c}} \\
(\mathrm{deg})\end{array}$ & $N_{0}{ }^{\mathrm{d}}$ & $N_{z}{ }^{\mathrm{e}}$ & Exposure Time $^{\mathrm{f}}$ \\
\hline \hline 20 & 2009 Apr 22 & 123748.36 & 620647.86 & 45 & 118 & 50 & $4 \times 1200 \mathrm{~s}$ \\
21 & 2009 Apr 23 & 123728.56 & 620947.81 & 45 & 100 & 35 & $3 \times 1200 \mathrm{~s}$ \\
22 & 2009 Apr 24 & 123602.88 & 621851.59 & 45 & 92 & 10 & $3 \times 1200 \mathrm{~s}$ \\
23 & 2010 Apr 18 & 123558.92 & 622021.56 & 45 & 107 & 55 & $3 \times 1140 \mathrm{~s}$ \\
\hline
\end{tabular}

NoTE. - The numbering of the DEIMOS slit masks begins at 20 to avoid any confusion with TKRS slit masks, which are numbered 1 through 18.

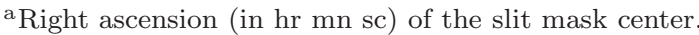

${ }^{\mathrm{b}}$ Declination (in deg min sec) of the slit mask center.

${ }^{\mathrm{c}}$ Position angle of the slit mask $(\mathrm{E}$ of $\mathrm{N})$; note that the orientation of individual slits vary.

${ }^{\mathrm{d}}$ Number of targets on slit mask.

dNumber of secure $(Q=-1,3,4)$ redshifts measured on slit mask.

$\mathrm{d}$ Total exposure time for slit mask (in seconds).

TABLE 2

REDSHIFT CATALOG

\begin{tabular}{cccccccccccccc}
\hline \hline Object ID $^{\mathrm{a}}$ & $\alpha^{\mathrm{b}}(\mathrm{J} 2000)$ & $\delta^{\mathrm{c}}(\mathrm{J} 2000)$ & $R_{\mathrm{AB}}{ }^{\mathrm{d}}$ & Mask $^{\mathrm{e}}$ & Slit $^{\mathrm{f}}$ & MJD $^{\mathrm{g}}$ & flag $^{\mathrm{h}}$ & $z^{\mathrm{i}}$ & $z_{\text {helio }^{\mathrm{j}}}$ & $Q^{\mathrm{k}}$ & $z_{\text {other }}{ }^{\mathrm{l}}$ & Ref $^{\mathrm{m}}$ \\
\hline 15018 & 189.20270 & 62.073785 & 22.05 & 20 & 000 & 54943.3 & 0 & 0.2856 & 0.2855 & 4 & $\ldots$ & $\ldots$ \\
26812 & 189.30411 & 62.174627 & 23.27 & 20 & 007 & 54943.3 & 0 & 0.8579 & 0.8579 & 4 & 0.8576 & 2 \\
27336 & 189.25811 & 62.155796 & 24.31 & 20 & 015 & 54943.3 & 0 & $\ldots$ & $\ldots$ & 2 & $\ldots$ & $\ldots$ \\
\hline
\end{tabular}

Note. - Table 2 is presented in its entirety in the electronic edition of the Journal. A portion is shown here for guidance regarding its form and content.

${ }^{\text {a } O b j e c t ~ i d e n t i f i c a t i o n ~ n u m b e r ~ i n ~} R$-band catalog of Capak et al. (2004).

${ }^{\mathrm{b}}$ Right ascension in decimal degrees from Capak et al. (2004).

${ }^{\mathrm{c}}$ Declination in decimal degrees from Capak et al. (2004).

${ }^{\mathrm{d}} R$-band magnitude in AB system from Capak et al. (2004).

eNumber of DEIMOS slit mask on which object was observed.

${ }^{\mathrm{f}}$ Number of slit on DEIMOS slit mask corresponding to object.

gModified Julian Date of observation.

${ }^{\mathrm{h}}$ Targeting Flag: $2=$ x-ray target, $1=$ MIPS target, $0=$ Main $R$-band selected target.

${ }^{\mathrm{i}}$ Redshift derived from observed spectrum.

${ }^{\mathrm{j}}$ Heliocentric-frame redshift.

${ }^{\mathrm{k}}$ Redshift quality code ( $\mathrm{star}=-1 ; \sim 90 \%$ confidence $=3 ; \sim 99 \%$ confidence $=4 ;$ unknown $=1,2$ ).

${ }^{1}$ Alternate redshift from literature.

${ }^{\mathrm{m}}$ Source of alternate redshift: (1) Wirth et al. (2004); (2) Barger et al. (2008).

MCC acknowledges support for this work provided by NASA through Hubble Fellowship grant \#HF-51269.01A awarded by the Space Telescope Science Institute, which is operated by the Association of Universities for Research in Astronomy, Inc., for NASA, under contract NAS 5-26555. This work was also supported in part by NSF grants AST-0808133, AST-0807630, and AST0806732 as well as Hubble Space Telescope Archival grant, HST-AR-10947.01. MCC thanks Greg Wirth and the entire Keck Observatory staff for their help in the acquisition of the DEEP3 Keck/DEIMOS data.

We also wish to recognize and acknowledge the highly significant cultural role and reverence that the summit of Mauna Kea has always had within the indigenous Hawaiian community. It is a privilege to be given the opportunity to conduct observations from this mountain.

Facilities: Keck:II (DEIMOS)

\section{REFERENCES}

Aird, J. et al. 2010, MNRAS, 401, 2531

Alexander, D. M. et al. 2003, AJ, 126, 539

Barger, A. J., Cowie, L. L., \& Wang, W. 2008, ApJ, 689, 687

Bertin, E. \& Arnouts, S. 1996, A\&AS, 117, 393

Capak, P. et al. 2004, AJ, 127, 180

Cohen, J. G., Hogg, D. W., Blandford, R., Cowie, L. L., Hu, E. Songaila, A., Shopbell, P., \& Richberg, K. 2000, ApJ, 538, 29

Cooper, M. C., Newman, J. A., Madgwick, D. S., Gerke, B. F., Yan, R., \& Davis, M. 2005, ApJ, 634, 833

Cooper, M. C. et al. 2006, MNRAS, 370, 198

Cowie, L. L., Barger, A. J., Hu, E. M., Capak, P., \& Songaila, A. 2004, AJ, 127, 3137
Davis, M. et al. 2003, in Society of Photo-Optical Instrumentation Engineers (SPIE) Conference Series, Vol. 4834, Society of PhotoOptical Instrumentation Engineers (SPIE) Conference Series, ed. P. Guhathakurta, 161-172

Davis, M. et al. 2007, ApJ, 660, L1

Dawson, S., Stern, D., Bunker, A. J., Spinrad, H., \& Dey, A. 2001, AJ, 122,598

Faber, S. M. et al. 2003, in Presented at the Society of Photo-Optical Instrumentation Engineers (SPIE) Conference, Vol. 4841, Society of Photo-Optical Instrumentation Engineers (SPIE) Conference Series, ed. M. Iye \& A. F. M. Moorwood, 1657-1669

Frayer, D. T. et al. 2006, ApJ, 647, L9

Giavalisco, M. et al. 2004, ApJ, 600, L93

Juneau, S. et al. 2010, submitted 
Kobulnicky, H. A. \& Kewley, L. J. 2004, ApJ, 617, 240

Laird, E. S. et al. 2009, ApJS, 180, 102

Lowenthal, J. D. et al. 1997, ApJ, 481, 673

Magnelli, B., Elbaz, D., Chary, R. R., Dickinson, M., Le Borgne, D., Frayer, D. T., \& Willmer, C. N. A. 2009, A\&A, 496, 57

- 2011, arXiv:1101.2467 [astro-ph]

Morrison, G. E., Owen, F. N., Dickinson, M., Ivison, R. J., \& Ibar, E. 2010, ApJS, 188, 178

Phillips, A. C., Guzman, R., Gallego, J., Koo, D. C., Lowenthal, J. D., Vogt, N. P., Faber, S. M., \& Illingworth, G. D. 1997, ApJ, 489,543
Reddy, N. A., Steidel, C. C., Erb, D. K., Shapley, A. E., \& Pettini, M. 2006, ApJ, 653, 1004

Riess, A. G. et al. 2007, ApJ, 659, 98

Treu, T. et al. 2005, ApJ, 633, 174

Weiner, B. J. et al. 2006, ApJ, 653, 1049

Wirth, G. D. et al. 2004, AJ, 127, 3121 